\title{
The Impact of Measurement Errors on ARMA Prediction
}

\author{
SERGIO G. KOREISHA* AND YUE FANG \\ University of Oregon, USA
}

\begin{abstract}
Measurement errors can have dramatic impact on the outcome of empirical analysis. In this article we quantify the effects that they can have on predictions generated from ARMA processes. Lower and upper bounds are derived for differences in minimum mean squared prediction errors (MMSE) for forecasts generated from data with and without errors. The impact that measurement errors have on MMSE and other relative measures of forecast accuracy are presented for a variety of model structures and parameterizations. Based on these results the need to set up the models in state space form to extract the signal component appears to depend upon whether processes are nearly non-invertible or non-stationary or whether the noise-to-signal ratio is very high. Copyright (C) 1999 John Wiley \& Sons, Ltd.
\end{abstract}

KEY WORDS autocovariance; forecasts; minimum mean square error

\section{INTRODUCTION}

Virtually all data contain observation errors. In addition, the measures often available to study economic and scientific phenomena are imperfect measurements of what is actually needed. The presence of measurement errors introduces added complexity in model identification since they will have an impact on the covariance structure of the observed data. Moreover, as demonstrated by Bell and Hilmer (1990) among others, parameter estimation is also very sensitive to the inclusion of error component factors in models.

Although much research has been conducted to deal with many of the problems associated with errors in variables, particularly in the area of parameter estimation or in the case of repeated time series measurements (Wong and Miller, 1990), as pointed in Wilcox (1992), most researchers do not spend much effort investigating the quality of the data, and often ignore measurement errors which may be important enough to influence the conclusions of empirical work as typically conducted.

\footnotetext{
* Correspondence to: Sergio G. Koreisha, Lundquist College of Business, University of Oregon, Eugene, OR 97403, USA. 
The presence of measurement errors naturally will also have an effect on predictability. Consider, for example, the following discrete-time system,

$$
y_{t}=x_{t}+\varepsilon_{t}
$$

where $y_{t}$ is the observed series, $x_{t}$ is a stationary and invertible ARMA signal process and the series $\varepsilon_{t}$, the error component, also referred to as the noise, is a sequence of uncorrelated random variables with mean zero and variance $\sigma_{\varepsilon}^{2}$, assumed to be independent to $x_{t}$. Then, for a given specific signal process $x_{t}$ with an ARMA $(p, q)$ structure, the observed series, $y_{t}$, will be identified as an $\operatorname{ARMA}(p, Q)$ process with $Q \leqslant \max (p, q)$, which may be different from the $\operatorname{ARMA}(p, q)$ structure of $\left\{x_{t}\right\}$ (Granger and Morris, 1976).

In the case that $q<p$, the possible misspecification may introduce prediction bias. If $q \geqslant p$, the observed series may be identified correctly as an $\operatorname{ARMA}(p, q)$ process, but the extra volatility from the measurement errors will distort the parameter estimation, and hence may cause problems with prediction particularly for small samples.

If a model without measurement errors is used in a situation where measurement errors are present, the $l$-step ahead prediction of $x_{t}$ based on the observed sequence $\left\{y_{t}\right\}$ is $\tilde{x}_{t}(l)=$ $E\left(y_{t+l} \mid y_{t}, y_{t-1}, \ldots\right)$. The impact of measurement errors on prediction can then be defined as the difference between the minimum mean squared predictive errors (MMSE) of $\tilde{x}_{t}(l)$ and $\hat{x}_{t}(l)$, where $\hat{x}_{t}(l)=E\left(x_{t+l} \mid x_{t}, x_{t-1}, \ldots\right)$ is the prediction based on the signal $\left\{x_{t}\right\}$. Denote this difference as $\triangle M M S E$, that is,

$$
\Delta M M S E=\operatorname{MMSE}\left(\tilde{x}_{t}(l)\right)-\operatorname{MMSE}\left(\hat{x}_{t}(l)\right)
$$

Consequently,

$$
\nabla M M S E \equiv \frac{\Delta M M S E}{\operatorname{MMSE}\left(\hat{x}_{t}(l)\right)}
$$

provides a measurement of the relative impact of measurement errors on MMSE predictions.

Our study will focus on the effects that $\left\{\varepsilon_{t}\right\}$ have on $\triangle M M S E$ and $\nabla M M S E$. The results obtained here should provide useful guidelines for evaluating predictions based on ARMA models when measurement errors are present but not treated explicitly.

The paper is organized as follows. In the next section we investigate the problem of making linear predictions when measurement errors are present, and discuss some of the properties of $\triangle M M S E$ and $\nabla M M S E$. We also derive lower and upper bounds for both $\triangle M M S E$ and $\nabla M M S E$ for stationary linear models, and show that the results are only dependent on the autocovariance structures, and not on the model specifications of $\left\{x_{t}\right\}$ and $\left\{y_{t}\right\}$. In the third section, we derive exact expressions for $\triangle M M S E$ and $\nabla M M S E$ for some low-order ARMA processes, and show that the impact of measurement errors on MMSE predictions is not significant unless the signal process, $\left\{x_{t}\right\}$, is nearly non-invertible or non-stationary. In the fourth section we extend our findings to seasonal ARMA processes. In the final section we offer some conclusions and directions for further research. 


\section{MMSE PREDICTION WITH MEASUREMENT ERRORS}

In this section we investigate the problem of predicting the value of $x_{t+l}$ in terms of $\left\{y_{i}\right\}_{i=1}^{t}$, and discuss the properties of $\triangle M M S E$ and $\nabla M M S E$. We will start by assuming that $\left\{x_{t}\right\}$ is a stationary linear process, and then proceed to develop lower and upper bounds for these measures of predictability when the signal process follows a stationary and invertible ARMA process, $\Phi(B) x_{t}=\Theta(B) a_{t}$, where $\Phi(B)$ and $\Theta(B)$ are finite polynomials in the backshift operator $B$ such that $B^{j} w_{t}=w_{t-j}$ and $\left\{a_{t}\right\}$ is a white noise process with variance $\sigma_{a}^{2}$. Such a signal process can also be expressed as autoregressive or moving average processes respectively, i.e. $\Pi(B) x_{t}=a_{t}$, or $x_{t}=\Psi(B) a_{t}$, where $\Pi=\Theta^{-1} \Phi$ and $\Psi=\Psi^{-1} \Theta$.

\section{MMSE predictors}

If measurement errors $\left\{\varepsilon_{t}\right\}$ are not present, the observed time series is simply $y_{t}=x_{t}$. The MMSE $l$-step ahead of prediction of $x_{t}$, based on a weighted average of previous observations and the forecasts made at previous lead times from the same origin, is defined in terms of the conditional expectation,

$$
\hat{x}_{t}(l)=E\left(x_{t+l} \mid y_{t}, y_{t-1}, \ldots, y_{1}\right)=E\left(x_{t+l} \mid x_{t}, x_{t-1}, \ldots, x_{1}\right)
$$

If we restrict ourselves to the class of linear predictors, then

$$
\hat{x}_{t}(l)=E\left(x_{t+l} \mid y_{t}, y_{t-1}, \ldots, y_{1}\right)=E\left(x_{t+l} \mid x_{t}, x_{t-1}, \ldots, x_{1}\right)=\sum_{i=1}^{t} \pi_{i, x}^{l} x_{i}
$$

where $\Pi_{x}^{l} \equiv\left(\pi_{1, x}^{l}, \pi_{2, x}^{l}, \ldots, \pi_{t, x}^{l}\right)^{\prime}$ is the weight vector estimated from the data which will yield the MMSE for $\hat{x}_{t}(l)$ (We have added the subscript $x$ to the $\Pi$ 's to emphasize that we are dealing with the series $\left\{x_{t}\right\}$. The theoretical expectation (5) requires knowledge of the $x_{i}^{\prime}$ 's going all the way back to the infinite past. However, because we have assumed that the model is invertible, the $\Pi$ weights in (5) form a convergent series. Consequently, for computational purposes, given a certain level of accuracy, the dependence on the distant past should be negligible.)

If the model contains measurement errors, the $l$-step ahead of linear prediction of $x_{t}, \tilde{x}_{t}(l)$, will be based on $\left\{y_{t}\right\}$, which will be distorted by the noise $\left\{\varepsilon_{t}\right\}$, and will be defined as

$$
\tilde{x}_{t}(l)=E\left(y_{t+l} \mid y_{t}, y_{t-1}, \ldots, y_{1}\right)=\sum_{i=1}^{t} \pi_{i, y}^{l} y_{i}
$$

Although the forecasts obtained from equation (6) are unbiased, as we shall show, $\triangle M M S E \neq 0$.

\section{Bounds for $\triangle M M S E$ and $\nabla M M S E$}

Let $X_{t}=\left(x_{t}, x_{t-1}, \ldots, x_{1}\right)^{\prime}, Y_{t}=\left(y_{t}, y_{t-1}, \ldots, y_{1}\right)^{\prime}, \mathcal{R}_{t, x}^{l}=\left(\gamma_{x}(l), \gamma_{x}(1+l), \ldots, \gamma_{x}(t-1-l)\right)^{\prime}$, where $\gamma_{x}(\cdot)$ is the autocovariance function of $x_{t}$, and $\Gamma_{t, x}=\left[\gamma_{x}(i-j)\right]_{i, j=1,2, \ldots, t}$. Then, the YuleWalker-type estimators for $\Pi_{x}^{l}$ are given by $\Pi_{x}^{l}=\Gamma_{t, x}^{-1} \mathcal{R}_{t, x}^{l}$. Thus,

$$
\hat{x}_{t}(l)=\left(\Pi_{x}^{l}\right)^{\prime} X_{t}=\left(\Gamma_{t, x}^{-1} \mathcal{R}_{t, x}^{l}\right)^{\prime} X_{t}
$$


and consequently, the MMSE of equation (7) becomes

$$
E\left(\hat{x}_{t}(l)-x_{t+l}\right)^{2}=\gamma_{x}(0)-\mathcal{R}_{t, x}^{l} \Gamma_{t, x}^{-1} \mathcal{R}_{t, x}^{l}
$$

(Corollary 5.1.1 in Brockwell and Davis, 1991).

Now let $\mathcal{R}_{t, y}^{l}, \Gamma_{t, y}$ and $\Pi_{y}^{l}$ be similarly defined for $y_{t}$. Hence, $\Pi_{y}^{l}=\Gamma_{t, y}^{-1} \mathcal{R}_{t, y}^{l}=\Gamma_{t, y}^{-1} \mathcal{R}_{t, x}^{l}$ since the autocovariance functions for $\left\{x_{t}\right\}$ and $\left\{y_{t}\right\}$ are the same. Moreover,

$$
\tilde{x}_{t}(l)=\left(\Pi_{y}^{l}\right)^{\prime} Y_{t}=\left(\Gamma_{t, y}^{-1} \mathcal{R}_{t, x}^{l}\right)^{\prime} Y_{t}
$$

and the MMSE of equation (9) can be calculated as follows:

$$
\begin{aligned}
E\left(\tilde{x}_{t}(l)-x_{t+l}\right)^{2} & =E\left(\tilde{x}_{t}(l)-y_{t+l}+\varepsilon_{t+l}\right)^{2} \\
& =E\left(\tilde{x}_{t}(l)-y_{t+l}\right)^{2}+2 E\left(\tilde{x}_{t}(l)-y_{t+l}\right) \varepsilon_{t+l}+E \varepsilon_{t+l}^{2} \\
& =\gamma_{y}(0)-\mathcal{R}_{t, y}^{l} \Gamma_{t, y}^{-1} \mathcal{R}_{t, y}^{l}-2 \sigma_{\varepsilon}^{2}+\sigma_{\varepsilon}^{2} \\
& =\gamma_{x}(0)+\sigma_{\varepsilon}^{2}-\mathcal{R}_{t, y}^{l}{ }^{\prime} \Gamma_{t, y}^{-1} \mathcal{R}_{t, y}^{l}-\sigma_{\varepsilon}^{2} \\
& =\gamma_{x}(0)-\mathcal{R}_{t, x}^{l}{ }^{\prime} \Gamma_{t, y}^{-1} \mathcal{R}_{t, x}^{l}
\end{aligned}
$$

Hence,

$$
\Delta M M S E=\mathcal{R}_{t, x}^{l}{ }^{\prime} \Gamma_{t, x}^{-1} \mathcal{R}_{t, x}^{l}-\mathcal{R}_{t, x}^{l}{ }^{\prime} \Gamma_{t, y}^{-1} \mathcal{R}_{t, x}^{l}
$$

and

$$
\nabla M M S E=\frac{\mathcal{R}_{t, x}^{l}{ }^{\prime} \Gamma_{t, x}^{-1} \mathcal{R}_{t, x}^{l}-\mathcal{R}_{t, x}^{l}{ }^{\prime} \Gamma_{t, y}^{-1} \mathcal{R}_{t, x}^{l}}{\operatorname{MMSE}(\hat{x}(l))}
$$

The conditions that $\gamma_{x}(0)>0$ and $\gamma_{x}(h) \rightarrow 0$ as $h \rightarrow \infty$ are sufficient to ensure that both $\Gamma_{t, x}$ and $\Gamma_{t, y}$ are non-singular for every $t$ (Proposition 5.1.1 in Brockwell and Davis, 1991). It should be noted that simplification of the matrix $\left[\Gamma_{t, x}^{-1}-\Gamma_{t, y}^{-1}\right]$ is, in general, very difficult, even though $\left[\Gamma_{t, y}-\Gamma_{t, x}\right]$ is of the form $\sigma_{\varepsilon}^{2} I$, where $I$ is a $t \times t$ identity matrix. The following theorem, which is the main result in this section, provides lower and upper bounds for $\triangle M M S E$ and $\nabla M M S E$.

Theorem 1 If $\Gamma_{t, x}$ is positive definite, then

$$
\frac{\sigma_{\varepsilon}^{2}}{\sigma_{\varepsilon}^{2}+\max _{i} \lambda_{i}} B_{1, t} \leqslant \Delta M M S E \leqslant \frac{\sigma_{\varepsilon}^{2}}{\sigma_{\varepsilon}^{2}+\min _{i} \lambda_{i}} B_{1, t}
$$

and

$$
\frac{\sigma_{\varepsilon}^{2}}{\sigma_{\varepsilon}^{2}+\max _{i} \lambda_{i}} B_{2, t} \leqslant \nabla M M S E \leqslant \frac{\sigma_{\varepsilon}^{2}}{\sigma_{\varepsilon}^{2}+\min _{i} \lambda_{i}} B_{2, t}
$$

where $\lambda_{i} s$ are eigenvalues of $\Gamma_{t, x}, B_{1, t}=\gamma_{x}(0)-\operatorname{MMSE}(\hat{x}(l))$ and $B_{2, t}=\left[\gamma_{x}(0)-\operatorname{MMSE}(\hat{x}(l))\right] /$ $\operatorname{MMSE}(\hat{x}(l))$.

The proof of the theorem is given in the Appendix. 
As can be seen, the bounds depend on $\sigma_{\varepsilon}^{2}$, and on the quality of the predictor $\hat{x}(l)$, which is measured by $B_{1, t}$ or $B_{2, t}$. In addition, the distribution of eigenvalues of $\Gamma_{t, x}$ also plays a critical role in the evaluation of $\triangle M M S E$ and $\nabla M M S E$. Furthermore, in the following sections, we will see that the eigenvalues of $\Gamma_{t, x}$ are closely related to process stationarity and invertibility.

In addition, the expected result that $\triangle M M S E \geqslant 0$ and $\nabla M M S E \geqslant 0$, which we state below as a corollary, can be easily verified, because $\gamma_{i}$ 's, $B_{1, t}$ and $B_{2, t}$ in equations (13) and (14) are nonnegative.

Corollary 1 If $\Gamma_{t, x}$ is positive definite, then

$$
\triangle M M S E \geqslant 0 \text { and } \nabla M M S E \geqslant 0
$$

As measurement errors decrease, $y_{t}$ converges to $x_{t}$, and the effects of measurement errors on predictions vanish. This result is a direct consequence of Theorem 1, which can be stated as follows:

Corollary 2 As $\sigma_{\varepsilon} \rightarrow 0$

$$
\triangle M M S E \rightarrow 0 \text { and } \nabla M M S E \rightarrow 0
$$

The results derived thus far are very general and depend only on the autocovariance structures. In the subsection below, we will assume that $\left\{x_{t}\right\}$ follows an ARMA process.

\section{Lower and upper bounds for some low-order ARMA processes}

The derivation of theoretical lower and upper bounds for $\triangle M M S E$ and $\nabla M M S E$ for general $\operatorname{ARMA}(p, q)$ models is very difficult and tedious. Here we provide derivations for just two processes-AR(1) and MA(1). Higher-order processes will be discussed in the following two sections. There we will obtain numerical values for both $\triangle M M S E$ and $\nabla M M S E$ based on some specific model parameterizations.

Example 1 Consider the case that $\left\{x_{t}\right\}$ follows an AR(1) process. Let $x_{t}=\phi x_{t-1}+a_{t}$ with $\sigma_{a}^{2}=1$ and define

$$
f(z)=\frac{1-\phi^{2}}{1-2 \phi \cos (\mathrm{z})+\phi^{2}}
$$

Then the smallest and largest eigenvalues of $\Gamma_{t, x}$ are

$$
\lambda_{\text {min }} \approx \frac{1}{1-\phi^{2}} \min _{z \in(0, \pi)} f(z)=\frac{1}{(1+|\phi|)^{2}}
$$

and

$$
\lambda_{\text {max }} \approx \frac{1}{1-\phi^{2}} \max _{z \in(0, \pi)} f(x)=\frac{1}{(1-|\phi|)^{2}}
$$


(Grenander and Szegö, 1958, see also Proposition 4.5.3 in Brockwell and Davis, 1991 for results on $\lambda_{\text {min }}$ and $\lambda_{\max }$ for more general ARMA processes). Hence, by Theorem 1,

$$
\frac{\sigma_{\varepsilon}^{2}}{\sigma_{\varepsilon}^{2}+\frac{1}{(1-|\phi|)^{2}}} B_{1, t} \leqslant \Delta M M S E \leqslant \frac{\sigma_{\varepsilon}^{2}}{\sigma_{\varepsilon}^{2}+\frac{1}{(1+|\phi|)^{2}}} B_{1, t}
$$

and

$$
\frac{\sigma_{\varepsilon}^{2}}{\sigma_{\varepsilon}^{2}+\frac{1}{(1-|\phi|)^{2}}} B_{2, t} \leqslant \Delta M M S E \leqslant \frac{\sigma_{\varepsilon}^{2}}{\sigma_{\varepsilon}^{2}+\frac{1}{(1+|\phi|)^{2}}} B_{2, t}
$$

In general, $B_{1, t} \geqslant B_{2, t}$ since $\operatorname{MMSE}\left(\hat{x}_{t}(l)\right) \geqslant \sigma_{a}^{2}=1$. If $l=1$, we have $B_{1, t}=B_{2, t}=\gamma_{x}(0)-$ $\operatorname{MMSE}\left(\hat{x}_{t}(1)\right)=\gamma_{x}(0)-\sigma_{a}^{2}=1 /\left(1-\phi^{2}\right)-1=\phi^{2} /\left(1-\phi^{2}\right)$.

When $|\phi|$ is small, equations (15) and (16) provide tight limits for $\triangle M M S E$ and $\nabla M M S E$. As $|\phi|$ goes to zero, the lower and upper bounds in equation (15) approach

$$
\frac{\sigma_{\varepsilon}^{2}}{\sigma_{\varepsilon}^{2}+1} B_{1, t}
$$

the lower and upper bounds in equation (16) approach

$$
\frac{\sigma_{\varepsilon}^{2}}{\sigma_{\varepsilon}^{2}+1} B_{2, t}
$$

When $\phi$ increases, the range between the lower and upper bounds increases. As $|\phi|$ approaches one, the lower bounds in equations (15) and (16) go to zero, while the upper bounds approach

$$
\frac{\sigma_{\varepsilon}^{2}}{\sigma_{\varepsilon}^{2}+0.25} B_{1, t}
$$

and

$$
\frac{\sigma_{\varepsilon}^{2}}{\sigma_{\varepsilon}^{2}+0.25} B_{2, t}
$$

respectively.

Example 2 In the second example, we assume that $\left\{x_{t}\right\}$ follows an MA(1) process. Let $x_{t}=$ $a_{t}-\theta a_{t-1}$ and $\sigma_{a}^{2}=1$. Then the eigenvalues of $\Gamma_{t, x}$ (see, Gregory and Karney, 1969) are

$$
\lambda_{i}=\gamma_{x}(0)+2 \gamma_{x}(1) \cos \left(\frac{k \pi}{n+1}\right) \quad k=1,2, \ldots, n
$$


where $\gamma_{x}(0)=\left(1+\theta^{2}\right)$ and $\gamma_{x}(1)=-\theta$. Hence, $\lambda_{\max } \approx(1+|\theta|)^{2}$ and $\lambda_{\min } \approx(1-|\theta|)^{2}$. Applying Theorem 1, we have

$$
\frac{\sigma_{\varepsilon}^{2}}{\sigma_{\varepsilon}^{2}+(1+|\theta|)^{2}} B_{1, t} \leqslant \Delta M M S E \leqslant \frac{\sigma_{\varepsilon}^{2}}{\sigma_{\varepsilon}^{2}+(1-|\theta|)^{2}} B_{1, t}
$$

and

$$
\frac{\sigma_{\varepsilon}^{2}}{\sigma_{\varepsilon}^{2}+(1+|\theta|)^{2}} B_{2, t} \leqslant \nabla M M S E \leqslant \frac{\sigma_{\varepsilon}^{2}}{\sigma_{\varepsilon}^{2}+(1-|\theta|)^{2}} B_{2, t}
$$

When $l=1, B_{1, t}=B_{2, t}=\gamma_{x}(0)-\operatorname{MMSE}\left(\hat{x}_{t}(1)\right)=\gamma_{x}(0)-\sigma_{a}^{2}=\left(1+\theta^{2}\right)-1=\theta^{2}$. If $l>1$, $B_{1, t}=B_{2, t}=0$ since $\operatorname{MMSE}\left(\hat{x}_{t}(l)\right)=\operatorname{MMSE}\left(\tilde{x}_{t}(l)\right)$.

When $l=1$, if $|\theta|$ is small, the range associated with equations (17) and (18) is very narrow. As $|\theta|$ goes to zero, the lower and upper bounds in these equations approach

$$
\frac{\sigma_{\varepsilon}^{2}}{\sigma_{\varepsilon}^{2}+1} \theta^{2}
$$

When $\theta$ increases, the difference between the lower and upper bounds increases. As $|\theta|$ goes to one, the lower and upper bounds in equations (17) and (18) approach

$$
\frac{\sigma_{\varepsilon}^{2}}{\sigma_{\varepsilon}^{2}+4} \theta^{2} \text { and } \theta^{2}
$$

respectively.

\section{Model misspecification and MMSE predictors}

Granger and Morris (1976) have proven that the sum of two uncorrelated processes, $\operatorname{ARMA}\left(p_{1}, q_{1}\right)$ and $\operatorname{ARMA}\left(p_{2}, q_{2}\right)$ is an $\operatorname{ARMA}\left(p^{*}, q^{*}\right)$ process, where $p^{*} \leqslant p_{1}+p_{2}$ and $q^{*} \leqslant \max \left(p_{1}+q_{2}, p_{2}+q_{1}\right)$. Thus, as pointed out earlier, given a specific ARMA $(p, q)$ signal process $\left\{x_{t}\right\}$, the observed series $\left\{y_{t}\right\}$ will be identified as an $\operatorname{ARMA}(p, Q)$ process, where $Q \leqslant \max (p, q)$.

If $q<p$, the number of past observations used in equations (5) and (6) to predict $x_{t+l}$ may be different because of the structural difference between $\Gamma_{t, x}^{-1}$ and $\Gamma_{t, y}^{-1}$. If $q \geqslant p$, the observed series will probably be correctly identified. However, the projection coefficients $\Pi_{t, x}^{l}$ and $\Pi_{t, y}^{l}$ will be different due to the impact of $\sigma_{\varepsilon}^{2}$ in $\Gamma_{t, y}^{l}$.

Example 3 Suppose that $\left\{x_{t}\right\}$ follows an $\mathrm{AR}(1)$ process, $x_{t}-\phi x_{t-1}=a_{t}$. If there is no measurement error, only the last observation $x_{t}$ is useful in predicting $x_{t+l}$. Therefore, the projection coefficients of $X_{t}, \Pi_{x}^{l}=(1,0,0, \ldots, 0)^{\prime}$ and $\hat{x}_{t}(l)=\phi^{l} x_{t}$. If the measurement errors are not zero, $y_{t}$ will follow an ARMA $(1,1)$ process. Consequently, $\pi_{i, y}^{l}$ will not be equal to zero for all $i=t, t-1, \ldots, 1$, hence the whole past history of $\left\{y_{t}\right\}$ will be used in the calculation of the MMSE prediction of $y_{t+l}$. The projection coefficients of $y_{t}$, i.e. $\pi_{i, y}^{l}$, approach zero asymptotically instead of cutting off abruptly.

Example 4 Assume that $\left\{x_{t}\right\}$ follows MA(1) process, that is $x_{t}=a_{t}-\theta_{1} a_{t-1}$ with $\sigma_{a}^{2}=1$. Then $y_{t}=x_{t}+\varepsilon_{t}$ will also follow an MA(1) process. Now assume that $y_{t}=b_{t}-\vartheta b_{t-1}$, where $\left\{b_{t}\right\}$ is a 
white noise process uncorrelated with $\left\{a_{t}\right\}$. The non-zero autocovariances of $y_{t}$ are given by $\gamma_{t}(0)=\left(1+\theta^{2}\right)+\sigma_{\varepsilon}^{2}=\left(1+\vartheta^{2}\right) \sigma_{b}^{2}$ and $\gamma_{y}(1)=-\theta=-\vartheta \sigma_{b}^{2}$. These two equations yield

$$
\left(1+\theta^{2}\right)+\sigma_{\varepsilon}^{2}=\left(1+\vartheta^{2}\right) \theta / \vartheta
$$

Using equation (19) one can easily show that $\vartheta$ and $\theta$ have equal sign and that $|\vartheta| \leqslant|\theta|$. This implies that the projection coefficients $\Pi_{y}^{l}$ and $\tilde{x}_{t}(l)$ are determined by an MA(1) process with $|\vartheta| \leqslant|\theta|$ and $\sigma_{b}^{2} \geqslant 1\left(=\sigma_{a}^{2}\right)$. Both $\Gamma_{t, x}$ and $\Gamma_{t, y}$ are tridiagonal matrices. A closed form of the inverse of tridiagonal matrices has been given by Gregory and Karney 1969). Thus, applying Gregory and Karney's result, one can find both $\Gamma_{t, x}^{-1}$ and $\Gamma_{t, y}^{-1}$.

\section{PREDICTIONS BASED ON ARMA MODELS}

In this section, we derive exact values for $\triangle M M S E$ and $\nabla M M S E$ associated with some low-order ARMA models. We have chosen model parameterizations of $\left\{x_{t}\right\}$ which not only conform to other previously published studies, such as Pukkila, Koreisha and Kallinen (1990) but which also cover a wide spectrum of parameter values ranging from well-defined stationary and invertible processes to nearly non-invertible or non-stationary processes. Without loss of generality, we will assume that the variance of $\left\{a_{t}\right\}, \sigma_{a}^{2}$, is one.

The assumption $\sigma_{a}^{2}=1$ implies that if $l=1, \operatorname{MMSE}(\hat{x}(l))=\sigma_{a}^{2}=1$. Hence, we have $\triangle M M S E=\nabla M M S E$ for the process without an MA part and $\triangle M M S E \approx \nabla M M S E$ for the process with an MA part. When $l>1, \triangle M M S E>\nabla M M S E$ since $\operatorname{MMSE}(\hat{x}(l))>1$.

To provide a measure of the magnitude of the measurement error relative to the signal process, let us define the noise-to-signal ratio as $\rho=\sigma_{\varepsilon} / \sigma_{x}$. In this study, we will evaluate the consequences on several-steps-ahead forecasts when $\rho$ will be set equal to $1 \%, 10 \%$ or $25 \%$. Since $\nabla M M S E$ is calculated in terms of second moments, we will compare $\nabla M M S E$ with $\rho^{2}$ instead of $\rho$. The corresponding $\rho^{2}$ values are $0.0001,0.01$ and 0.0625 , respectively. These three levels of $\rho$ ( or $\rho^{2}$ ) cover a wide range of situations usually encountered in practice (Bell and Wilcox, 1993).

It should be noted that if $x_{t}$ is an $\operatorname{AR}(p)$ process, $\nabla M M S E$ does not depend on the sample size $t$ as long as $t>p$. This is because the only last $p$ observations are used for prediction. On the other hand, if $x_{t}$ has an MA component, $\nabla M M S E$ does depend on $t$ since all past observations are required to calculate the MMSE prediction. However, these statistics are not noticeably affected by the sample size, especially when roots of the polynomials in equation (4) are not near the unit circle. Hence, for brevity, we will report results only for $t=100$. For illustrative purposes, however, we will also provide rests on $t=400$ for MA(1) structures.

Tables I-V contain $\nabla M M S E$ 's for some of the low-order $\operatorname{ARMA}(p, q)$ structures we studied for several prediction horizons, $l$, and levels of $\rho^{2}$. (For brevity we have omitted the results associated with $\triangle M M S E$ for most of the $\operatorname{ARMA}(p, q)$ structures in these tables. Table IV, however, contrasts $\triangle M M S E$ and $\nabla M M S E$ for various ARMA(1,1) parameterizations). In general we see that the impact that measurement errors have on MMSE predictions depends mainly on the invertibility and stationary properties of the $\left\{x_{t}\right\}$ processes, and naturally, on the magnitude of $\rho^{2}$. Unless the roots of the characteristic polynomials are near the unit circle, the impact is relatively negligible and concentrated primarily around the first few-steps-ahead forecasts. When the processes are nearly non-stationary, as exemplified by the AR(1) process with $|\phi| \geqslant 0.99$ and the $\mathrm{AR}(2)$ process with $\phi_{1}=1.8$, and $\phi_{2}=-0.9$, the impact of measurement 
Table I. $\nabla M M S E$ of $\mathrm{AR}(1)$ and $\mathrm{AR}(2)$ processes for several forecast horizons and different levels of measurement errors

\begin{tabular}{|c|c|c|c|c|c|c|c|c|c|}
\hline \multirow[b]{2}{*}{$\rho^{2}$} & \multirow[b]{2}{*}{$l$} & \multicolumn{4}{|c|}{$\operatorname{AR}(1): \phi_{1}$} & \multicolumn{4}{|c|}{$\operatorname{AR}(2):\left(\phi_{1}, \phi_{2}\right)$} \\
\hline & & $\begin{array}{c}0.30 \text { or } \\
-0.30\end{array}$ & $\begin{array}{c}0.50 \text { or } \\
-0.50\end{array}$ & $\begin{array}{c}0.90 \text { or } \\
-0.90\end{array}$ & $\begin{array}{c}0.99 \text { or } \\
-0.99\end{array}$ & $\begin{array}{c}(1.42, \\
-0.73)\end{array}$ & $\begin{array}{c}(1.80 \\
-0.90)\end{array}$ & $\begin{array}{l}(0.50) \\
0.30)\end{array}$ & $\begin{array}{c}(-0.30 \\
0.50)\end{array}$ \\
\hline \multirow[t]{4}{*}{0.0001} & 1 & $9.889 \mathrm{e}-06$ & $3.333 \mathrm{e}-05$ & 0.0004261 & 0.0049006 & 0.0016699 & 0.0204784 & $2.627 \mathrm{e}-05$ & $7.082 \mathrm{e}-05$ \\
\hline & 2 & $8.165 \mathrm{e}-07$ & $6.666 \mathrm{e}-06$ & 0.0001907 & 0.0024257 & 0.0005925 & 0.0096284 & $5.832 \mathrm{e}-05$ & $7.082 \mathrm{e}-05$ \\
\hline & 5 & $5.904 \mathrm{e}-10$ & $9.774 \mathrm{e}-08$ & $5.351 \mathrm{e}-05$ & 0.0009411 & $1.457 \mathrm{e}-05$ & 0.0022898 & $1.350 \mathrm{e}-05$ & $1.444 \mathrm{e}-05$ \\
\hline & 10 & $3.486 \mathrm{e}-15$ & $9.535 \mathrm{e}-11$ & $1.383 \mathrm{e}-05$ & 0.0004469 & $2.075 \mathrm{e}-06$ & $8.338 \mathrm{e}-05$ & $2.390 \mathrm{e}-06$ & $3.376 \mathrm{e}-06$ \\
\hline \multirow[t]{4}{*}{0.01} & 1 & 0.0009783 & 0.0032896 & 0.0405791 & 0.3596025 & 0.1455287 & 1.1615970 & 0.0075236 & 0.0069681 \\
\hline & 2 & $8.077 \mathrm{e}-05$ & 0.0006579 & 0.0181597 & 0.1779943 & 0.0499810 & 0.4802442 & 0.0057359 & 0.0069593 \\
\hline & 5 & $5.841 \mathrm{e}-08$ & $9.647 \mathrm{e}-06$ & 0.0050957 & 0.0690587 & 0.0014318 & 0.0984472 & 0.0013287 & 0.0014225 \\
\hline & 10 & $3.449 \mathrm{e}-13$ & $9.412 \mathrm{e}-09$ & 0.0013174 & 0.0327956 & 0.0001962 & 0.0059951 & 0.0002332 & 0.0003324 \\
\hline \multirow[t]{4}{*}{0.0625} & 1 & 0.0057862 & 0.0192588 & 0.2094753 & 1.3018980 & 0.6219203 & 3.8795890 & 0.0439953 & 0.0402842 \\
\hline & 2 & 0.0004778 & 0.0038518 & 0.0937431 & 0.6444068 & 0.1951217 & 1.3721710 & 0.0330805 & 0.0399863 \\
\hline & 5 & $3.455 \mathrm{e}-07$ & $5.648 \mathrm{e}-05$ & 0.0263046 & 0.2500185 & 0.0083004 & 0.2300115 & 0.0077032 & 0.0082634 \\
\hline & 10 & $2.040 \mathrm{e}-12$ & $5.510 \mathrm{e}-08$ & 0.0068006 & 0.1187326 & 0.0010888 & 0.0252748 & 0.0013516 & 0.0019278 \\
\hline
\end{tabular}

Notes:

(1) The observations follow $y_{t}=x_{t}+\varepsilon_{t}$.

(2) In the $\operatorname{AR}(1)$ case, $x_{t}-\phi_{1} x_{t-1}=a_{t}$ with $\sigma_{a}^{2}=1$ and

$$
\sigma_{\varepsilon}^{2}=\rho^{2} \frac{1}{1-\phi_{1}^{2}} .
$$

(3) In the $\operatorname{AR}(2)$ case, $x_{t}-\phi_{1} x_{t-1}-\phi_{2} x_{t-2}=a_{t}$ with $\sigma_{a}^{2}=1$ and

$$
\sigma_{\varepsilon}^{2}=\rho^{2} \frac{1-\phi_{2}}{\left(1+\phi_{2}\right)\left(\left(1-\phi_{2}\right)^{2}-\phi_{1}^{2}\right)} .
$$

(4) The result does not depend on the sample size $t$; results shown here are for $t=100$ observations.

errors as measured by $\nabla M M S E$ for the first-step-ahead forecast can be quite dramatic: $36 \%$ when $\rho=10 \%$, and $130 \%$ when $\rho=25 \%$ for the AR(1) process; and $116 \%$ when $\rho=10 \%$, and $388 \%$ when $\rho=25 \%$ for the $\operatorname{AR}(2)$ process. Note, however, how small the impact becomes, even for these processes, when the forecast horizon increases to ten periods: $3.28 \%$ and $11.9 \%$ for the AR(1) process, and $0.6 \%$ and $2.53 \%$ for the $\operatorname{AR}(2)$ process, when $\rho=10 \%$ and $25 \%$, respectively. For pure MA processes the impact of measurement errors are only felt for forecast horizons $l \leqslant q$ since $\gamma_{x}(l)$ is zero for $l>q$. As can be seen, the impact of measurement errors in the model prediction follows a pattern very similar to pure AR processes, more noticeably when the processes are nearly non-invertible, but they are generally of lower magnitude.

For mixed processes the impact also appears to be dependent on model parameterization. Those structures which when converted into equivalent $\operatorname{AR}(\infty)$ representations have $\pi$-weights which go to zero relatively slowly as the lag length increases, feel more of the impact of measurement errors than those for which the $\pi$-weights go to zero rapidly. Note, for example, when $l=1$, how large is the difference in $\nabla M M S E$ for $\rho=10 \%$ and $\rho=25 \%$, respectively for ARMA(1,1) processes with $\phi=0.8, \theta=0.7\left(\pi_{1}=0.1, \pi_{2}=0.1 \times 0.7, \pi_{3}=0.1 \times 0.7^{2}, \ldots\right)$ and with $\phi=0.8$ and $\theta=-0.7\left(\pi_{1}=1.5, \pi_{2}=1.5 \times 0.7, \pi_{3}=1.5 \times 0.7^{2}, \ldots\right)$. The impact of the measurement errors also decreases dramatically as the forecast horizon increases. For the 
Table II. $\nabla M M S E$ of MA(1) process for several forecast horizons and different levels of measurement errors $(t=100$ and $t=400)$

\begin{tabular}{|c|c|c|c|c|c|c|c|c|c|}
\hline \multirow[b]{3}{*}{$\rho^{2}$} & \multirow[b]{3}{*}{$l$} & \multicolumn{8}{|c|}{$\theta_{1}$} \\
\hline & & \multicolumn{2}{|c|}{0.30 or -0.30} & \multicolumn{2}{|c|}{0.50 or -0.05} & \multicolumn{2}{|c|}{0.90 or -0.90} & \multicolumn{2}{|c|}{0.99 or -0.99} \\
\hline & & $t=100$ & $t=400$ & $t=100$ & $t=400$ & $t=100$ & $t=400$ & $t=100$ & $t=400$ \\
\hline 0.0001 & $\begin{array}{c}1 \\
2,5,10\end{array}$ & $\begin{array}{c}1.078 \mathrm{e}-05 \\
0\end{array}$ & $\begin{array}{c}1.078 \mathrm{e}-05 \\
0\end{array}$ & $\begin{array}{c}4.166 \mathrm{e}-05 \\
0\end{array}$ & $\begin{array}{c}4.166 \mathrm{e}-05 \\
0\end{array}$ & $\begin{array}{c}0.0007678 \\
0\end{array}$ & $\begin{array}{c}0.0007678 \\
0\end{array}$ & $\begin{array}{c}0.0051690 \\
0\end{array}$ & $\begin{array}{c}0.0071218 \\
0\end{array}$ \\
\hline 0.01 & $\begin{array}{c}1 \\
2,5,10\end{array}$ & $\begin{array}{c}0.0010640 \\
0\end{array}$ & $\begin{array}{c}0.0010640 \\
0\end{array}$ & $\begin{array}{c}0.0040766 \\
0\end{array}$ & $\begin{array}{c}0.0040766 \\
0\end{array}$ & $\begin{array}{c}0.0555977 \\
0\end{array}$ & $\begin{array}{c}0.0559775 \\
0\end{array}$ & $\begin{array}{c}0.1175039 \\
0\end{array}$ & $\begin{array}{c}0.1208585 \\
0\end{array}$ \\
\hline 0.0625 & $\begin{array}{c}1 \\
2,5,10\end{array}$ & $\begin{array}{c}0.0062287 \\
0\end{array}$ & $\begin{array}{c}0.0062287 \\
0\end{array}$ & $\begin{array}{c}0.0229490 \\
0\end{array}$ & $\begin{array}{c}0.0229490 \\
0\end{array}$ & $\begin{array}{c}0.1869673 \\
0\end{array}$ & $\begin{array}{c}0.1869673 \\
0\end{array}$ & $\begin{array}{c}0.2799237 \\
0\end{array}$ & $\begin{array}{c}0.2837658 \\
0\end{array}$ \\
\hline
\end{tabular}

Notes:

(1) The signal is an MA(1) process $x_{t}=a_{t}-\theta_{1} a_{t-1}$ with $\sigma_{a}^{2}=1$ and the observations follow $y_{t}=x_{t}+\varepsilon_{t}$.

(2) $\sigma_{\varepsilon}^{2}=\rho^{2}\left(1+\theta_{1}^{2}\right)$.

(3) The sample size effects are negligible, especially when the signal process is not nearly non-invertible. When the signal process is nearly non-invertible $\left(\left|\theta_{1}\right| \geqslant 0.9\right)$, some of $\nabla M M S E$ s at $t=400$ are greater than those at $t=100$ due to the different decay speeds of $\operatorname{MMSE}\left(\tilde{x}_{t}(l)\right)$ and $\operatorname{MMSE}\left(\hat{x}_{t}(l)\right)$. For example, when $\left|\theta_{1}\right|=0.99, \operatorname{MMSE}\left(\tilde{x}_{t}(1)\right)=$ 1.008193 and $\operatorname{MMSE}\left(\hat{x}_{t}(1)\right)=1.003008$ and for $t=100$, and $\operatorname{MMSE}\left(\tilde{x}_{t}(1)\right)=1.007128$ and $\operatorname{MMSE}\left(\hat{x}_{t}(1)\right)=$ 1.000006 for $t=400$.

ARMA(1,2) structure with $\phi_{1}=-0.8, \theta_{1}=1.4$ and $\theta_{2}=-0.6$ for $\rho=10 \%, \nabla M M S E$ changes from $115 \%$ when $l=1$ to $0.22 \%$ when $l=10$; for the $\operatorname{ARMA}(2,1)$ with $\phi_{1}=-0.5, \phi_{2}=-0.9$ and $\theta=0.6$, the corresponding values when $\rho=25 \%$ are $87 \%$ and $4.1 \%$ for $l=1$ and 10 respectively.

Finally, as mentioned earlier, sample size does not have a noticeable effect on the prediction measures as can be seen by contrasting the values of these measures for $t=100$ and $t=400$ in Table II.

\section{SEASONAL ARMA PROCESSES}

Seasonal models can be viewed as special forms of the ARMA models. Results for low-order non-seasonal ARMA processes in the previous sections, as we shall demonstrate, can be used to show the impact that measurement errors have on the predictability of seasonal models. To illustrate how one can apply the results for low-order non-seasonal ARMA models to seasonal ARMA processes, we will consider two often used multiplicative structures.

Example 5 Suppose that $x_{t}$ is an $\operatorname{ARMA}(0,1) \times \operatorname{SARMA}(1,0)_{12}$ process, that is $x_{t}=\Phi x_{t-12}$ $+a_{t}-\theta a_{t-1}$. Then $\gamma_{x}(0)=\left(1+\theta^{2}\right) /\left(1-\Phi^{2}\right), \gamma_{x}(12 k)=\Phi^{k} \gamma_{x}(0)$, and $\gamma_{x}(12 k-1)=\gamma_{x}(12 k+$ $1)=-\theta \Phi^{k} \gamma_{x}(0) /\left(1+\theta^{2}\right)$ for $k=1,2, \ldots, \gamma_{x}(\cdot)=0$ at all other lags. Define the $\operatorname{ARMA}(1,1)$ process $\left\{x_{t}^{*}\right\}$ as $x_{t}^{*}=\Phi x_{t-1}^{*}+a_{t}-\theta a_{t-1}$. If we compare the covariances of $\left\{x_{t}\right\}$ with those of $\left\{x_{t}^{*}\right\}$, we see that

$$
\operatorname{MMSE}\left(\hat{x}_{t}(l)\right)=\operatorname{MMSE}\left(\hat{x}_{t}^{*}\left(l^{*}\right)\right) \quad \text { for } 12\left(l^{*}-1\right)<l \leqslant 12 l^{*}, \quad l^{*}=1,2,3, \ldots
$$


Table III. $\nabla M M S E$ of MA(2) and MA(3) processes for several forecast horizons and different levels of measurement errors

\begin{tabular}{|c|c|c|c|c|c|c|c|c|c|}
\hline \multirow[b]{2}{*}{$\rho^{2}$} & \multirow[b]{2}{*}{$l$} & \multicolumn{4}{|c|}{$\operatorname{MA}(2):\left(\theta_{1}, \theta_{2}\right)$} & \multicolumn{4}{|c|}{$\operatorname{MA}(3):\left(\theta_{1}, \theta_{2}, \theta_{3}\right)$} \\
\hline & & $\begin{array}{c}(1.42, \\
-0.73)\end{array}$ & $\begin{array}{l}(1.8, \\
-0.9)\end{array}$ & $(0.5,0.3)$ & $\begin{array}{c}(-0.3 \\
0.5)\end{array}$ & $\begin{array}{c}(1.0,0.8 \\
-0.9)\end{array}$ & $\begin{array}{c}(0.5,0.3 \\
-0.3)\end{array}$ & $\begin{array}{c}(0.8,0.8 \\
-0.64)\end{array}$ & $\begin{array}{l}(-0.4 \\
-0.55 \\
-0.22)\end{array}$ \\
\hline \multirow[t]{5}{*}{0.0001} & 1 & 0.0019661 & 0.0228484 & 0.0001665 & 0.001450 & 0.0211620 & $8.482 \mathrm{e}-05$ & 0.0058830 & $6.506 \mathrm{e}-05$ \\
\hline & 2 & 0.0004091 & 0.0043595 & $2.163 \mathrm{e}-05$ & $6.398 \mathrm{e}-05$ & 0.0007031 & $1.511 \mathrm{e}-05$ & 0.0002882 & $5.647 \mathrm{e}-05$ \\
\hline & 3 & 0 & 0 & 0 & 0 & 0.0064248 & $1.530 \mathrm{e}-05$ & 0.0010984 & $7.152 \mathrm{e}-06$ \\
\hline & 5 & 0 & 0 & 0 & 0 & 0 & 0 & 0 & 0 \\
\hline & 10 & 0 & 0 & 0 & 0 & 0 & 0 & 0 & 0 \\
\hline \multirow[t]{5}{*}{0.01} & 1 & 0.1495970 & 0.5493175 & 0.0150871 & 0.0131661 & 0.3621351 & 0.0082420 & 0.1970614 & 0.0063627 \\
\hline & 2 & 0.0275923 & 0.0716249 & 0.0019943 & 0.0059355 & 0.0290324 & 0.0014899 & 0.0218090 & 0.0054987 \\
\hline & 3 & 0 & 0 & 0 & 0 & 0.0866441 & 0.0014806 & 0.0328719 & 0.0006956 \\
\hline & 5 & 0 & 0 & 0 & 0 & 0 & 0 & 0 & 0 \\
\hline & 10 & 0 & 0 & 0 & 0 & 0 & 0 & 0 & 0 \\
\hline \multirow[t]{5}{*}{0.0625} & 1 & 0.5269499 & 1.2148580 & 0.0670375 & 0.0589886 & 0.7721829 & 0.0450623 & 0.4749465 & 0.0358288 \\
\hline & 2 & 0.0756443 & 0.1155425 & 0.0094341 & 0.0286489 & 0.1181814 & 0.0087035 & 0.0947855 & 0.0303103 \\
\hline & 3 & 0 & 0 & 0 & 0 & 0.1515817 & 0.0079593 & 0.0703101 & 0.0038140 \\
\hline & 5 & 0 & 0 & 0 & 0 & 0 & 0 & 0 & 0 \\
\hline & 10 & 0 & 0 & 0 & 0 & 0 & 0 & 0 & 0 \\
\hline
\end{tabular}

Notes:

(1) The observations follow $y_{t}=x_{t}+\varepsilon_{t}$

(2) For the MA(2) case, $x_{t}=a_{t}-\theta_{1} a_{t-1}-\theta_{2} a_{t-2}$ with $\sigma_{a}^{2}=1$ and $\sigma_{\varepsilon}^{2}=\rho^{2}\left(1+\theta_{1}^{2}+\theta_{2}^{2}\right)$.

(3) For the MA(3) case, $x_{t}=a_{t}-\theta_{1} a_{t-1}-\theta_{2} a_{t-2}-\theta_{3} a_{t-3}$ with $\sigma_{\varepsilon}^{2} \stackrel{\varepsilon}{=} 1$ and $\sigma_{\varepsilon}^{2}=\rho^{2}\left(1+\theta_{1}^{2}+\theta_{2}^{2}+\theta_{3}^{2}\right)$.

(4) The result does not depend on the sample size $t=100$ observations.

where $\hat{x}_{t}(l)$ is the MMSE predictor of $x_{t}(t+l)$ based on $\left\{x_{t}\right\}$ and $\hat{x}_{t}^{*}\left(l^{*}\right)$ is the MMSE predictor of $x_{t}^{*}\left(t+l^{*}\right)$ based on $\left\{x_{t}^{*}\right\}$. A similar relationship exists for $\operatorname{MMSE}\left(\tilde{x}_{t}(l)\right)$ and $\operatorname{MMSE}\left(\tilde{x}_{t}^{*}\left(l^{*}\right)\right)$, where $\tilde{x}_{t}(l)$ is the MMSE predictor of $y_{t}(t+l)$ based on $\left\{y_{t}\right\}$ and $\tilde{x}_{t}^{*}(l)$ is the MMSR predictor of $y_{t}^{*}\left(t+l^{*}\right)$ based on $y_{t}^{*} \equiv x_{t}^{*}+\varepsilon_{t}$, namely,

$$
\operatorname{MMSE}\left(\tilde{x}_{t}(l)\right)=\operatorname{MMSE}\left(\tilde{x}_{t}^{*}\left(l^{*}\right)\right) \quad \text { for } 12\left(l^{*}-1\right)<l \leqslant 12 l^{*}, \quad l^{*}=1,2,3, \ldots
$$

Therefore, in order to analyze the impact of measurement errors on MMSE predictions for $\operatorname{ARMA}(0,1) \times \operatorname{SARMA}(1,0)_{12}$, one only needs to evaluate the corresponding statistics for the $\operatorname{ARMA}(1,1)$ process, $\left\{x_{t}^{*}\right\}$.

Example 6 Airline Model (The actual airline passenger model has an integrated component (see Box and Jenkins, 1976, p. 531.)) Suppose that $x_{t}$ follows an ARMA $(0,1) \times \operatorname{SARMA}(0,1)_{12}$, that is, $x_{t}=a_{t}-\theta a_{t-1}-\Theta a_{t-12}+\theta \Theta a_{t-12-1}$. If $\sigma_{a}^{2}=1$, the autocovariance function for $x_{t}$ is $\gamma_{x}(0)=\left(1+\theta^{2}\right)\left(1+\Theta^{2}\right), \quad \gamma_{x}(1)=\theta\left(1+\Theta^{2}\right), \quad \gamma_{x}(k)=0 \quad$ for $\quad k=2,3, \ldots, 12-2$, $\gamma_{x}(12-1)=\theta \Theta, \gamma_{x}(12)=\Theta\left(1+\theta^{2}\right), \gamma_{x}(12+1)=\theta \Theta$, and $\gamma_{x}(l)=0$ for $l \geqslant 12+2$. 
Table IV. $\triangle M M S E$ and $\nabla M M S E$ of ARMA $(1,1)$ process for several forecast horizons and different levels of measurement errors

\begin{tabular}{|c|c|c|c|c|c|c|c|c|c|}
\hline \multirow[b]{3}{*}{$\rho^{2}$} & \multirow[b]{3}{*}{$l$} & \multicolumn{8}{|c|}{$\left(\phi_{1}, \theta_{2}\right)$} \\
\hline & & \multicolumn{2}{|c|}{$\begin{array}{l}(0.8,-0.7) \text { or } \\
\quad(-0.8,0.7)\end{array}$} & \multicolumn{2}{|c|}{$\begin{array}{l}(0.8,0.7) \text { or } \\
(-0.8,-0.7)\end{array}$} & \multicolumn{2}{|c|}{$\begin{array}{c}(0.9,-0.9) \text { or } \\
(-0.9,0.9)\end{array}$} & \multicolumn{2}{|c|}{$\begin{array}{l}(0.3,0.5) \text { or } \\
(-0.3,-0.5)\end{array}$} \\
\hline & & $\triangle M M S E$ & $\nabla M M S E$ & $\Delta M M S E$ & $\nabla M M S E$ & $\Delta M M S E$ & $\nabla M M S E$ & $\Delta M M S E$ & $\nabla M M S E$ \\
\hline \multirow[t]{4}{*}{0.0001} & 1 & 0.0031771 & 0.0031771 & $2.015 \mathrm{e}-06$ & $2.015 \mathrm{e}-06$ & $2.015 \mathrm{e}-06$ & 0.0269167 & $5.567 \mathrm{e}-06$ & $5.567 \mathrm{e}-06$ \\
\hline & 2 & 0.0020334 & 0.0006256 & $1.290 \mathrm{e}-06$ & $1.277 \mathrm{e}-06$ & 0.0218025 & 0.0051421 & $5.010 \mathrm{e}-07$ & $4.818 \mathrm{e}-07$ \\
\hline & 5 & 0.0005330 & $8.595 \mathrm{e}-05$ & $3.381 \mathrm{e}-07$ & $3.304 \mathrm{e}-07$ & 0.0115868 & 0.0010817 & $3.653 \mathrm{e}-10$ & $3.499 \mathrm{e}-10$ \\
\hline & 10 & $5.723 e-05$ & $8.109 \mathrm{e}-06$ & $3.630 \mathrm{e}-08$ & $3.534 \mathrm{e}-08$ & 0.0040401 & 0.0002608 & $2.157 \mathrm{e}-15$ & $2.066 \mathrm{e}-15$ \\
\hline \multirow[t]{4}{*}{0.01} & 1 & 0.2166930 & 0.2166930 & 0.0002000 & 0.0002000 & 0.6608152 & 0.6608119 & 0.0005494 & 0.0005494 \\
\hline & 2 & 0.1386835 & 0.0426719 & 0.0001280 & 0.0001267 & 0.5352603 & 0.1262405 & $4.945 \mathrm{e}-05$ & $4.755 \mathrm{e}-05$ \\
\hline & 5 & 0.0363551 & 0.0058624 & $3.355 \mathrm{e}-05$ & $3.280 \mathrm{e}-05$ & 0.2844593 & 0.0265551 & $3.605 \mathrm{e}-08$ & $3.453 \mathrm{e}-08$ \\
\hline & 10 & 0.0039036 & 0.0005469 & $3.603 \mathrm{e}-06$ & $3.507 \mathrm{e}-06$ & 0.0991848 & 0.0064019 & $2.129 \mathrm{e}-13$ & $2.039 \mathrm{e}-13$ \\
\hline \multirow[t]{4}{*}{0.0625} & 1 & 0.7277709 & 0.7277709 & 0.0012022 & 0.0012022 & 1.7204460 & 1.7204370 & 0.0032118 & 0.0032118 \\
\hline & 2 & 0.4657734 & 0.1433149 & 0.0007694 & 0.0007618 & 1.3935610 & 0.3286697 & 0.0002891 & 0.0002779 \\
\hline & 5 & 0.1220997 & 0.0196890 & 0.0002017 & 0.0001971 & 0.7405955 & 0.0691368 & $2.107 \mathrm{e}-07$ & $2.019 \mathrm{e}-07$ \\
\hline & 10 & 0.0131104 & 0.0018369 & $2.166 \mathrm{e}-05$ & $2.108 \mathrm{e}-05$ & 0.2582297 & 0.0166674 & $1.244 \mathrm{e}-12$ & $1.192 \mathrm{e}-12$ \\
\hline
\end{tabular}

Notes:

(1) The signal is an $\operatorname{ARMA}(1,1)$ process $x_{t}-\phi_{1} x_{t-1}=a_{t}-\theta_{1} a_{t-1}$ with $\sigma_{a}^{2}=1$ and the observations follow (2) $y_{t}=x_{t}+\varepsilon_{2}$.

$$
\sigma_{\varepsilon}^{2}=\rho^{2} \frac{1+\theta_{1}^{2}-2 \phi_{1} \theta_{1}}{1-\phi_{1}^{2}}
$$

(3) The result does not depend on the sample size $t$; results shown here are for $t=100$ observations.

Let $x_{t}^{*} \equiv a_{t}-\theta a_{t-1}-\Theta a_{t-2}+\theta \Theta a_{t-3}$. Furthermore, let $\hat{x}_{t}(l)$ be the MMSE predictor of $x_{t}(t+l)$ based on $\left\{x_{t}\right\}$, and $\hat{x}_{t}^{*}\left(l^{*}\right)$ be the MMSE predictor of $x_{t}^{*}\left(t+l^{*}\right)$ based on $\left\{x_{t}^{*}\right\}$. Following a similar line of logic as in the previous example, we have

$$
\operatorname{MMSE}\left(\hat{x}_{t}(l)=\operatorname{MMSE}\left(\hat{x}_{t}^{*}\left(l^{*}\right)\right)\right.
$$

for $l^{*}=1$ if $l=1 ; l^{*}=2$ if $l=2,3, \ldots, 12 ; l^{*}=3$ if $l=12+1$; and $l^{*}>3$ if $l>12+1$. Equation (22) holds for $\operatorname{MMSE}\left(\tilde{x}_{t}(l)\right)$ and $\operatorname{MMSE}\left(\tilde{x}_{t}^{*}\left(l^{*}\right)\right)$, where $\tilde{x}_{t}(l)$ is the MMSE predictor of $y_{t}(t+l)$ based on $\left\{y_{t}\right\}$ and $\tilde{x}_{t}^{*}(l)$ is the MMSE predictor of $y_{t}^{*}\left(t+l^{*}\right)$ based on $y_{t}^{*} \equiv x_{t}^{*}+\varepsilon_{t}$.

In examining the results, for instance, for the MA(3) model in Table III with $\theta_{1}=-0.40$, $\theta_{2}=-0.55$ and $\theta_{3}=-0.22$ (equivalent to the $w_{t}=(1+0.4 B)\left(1+0.55 B^{12}\right) a_{t}$ parameterization obtained by Box and Jenkins, 1976 for the airline data), we see that the impact of measurement errors regardless of the value of $\rho$ is rather negligible. For equivalent seasonal processes with eigenvalues nearer the unit circle we observe the same type of behaviour noted earlier for nonseasonal processes, namely that their impact decreases as the forecast horizon increases. Note, for example, how dramatic are the changes in $\nabla M M S E$ for the parameterization $\theta_{1}=1.0, \theta_{2}=0.8$ and $\theta_{3}=-0.9$ (equivalent to the MA(13) model with only three non-zero parameters $\theta_{1}, \theta_{12}$, 
Table V. $\nabla M M S E$ of $\operatorname{ARMA}(1,2)$ and ARMA $(2,1)$ processes for several forecast horizons and different levels of measurement errors

\begin{tabular}{|c|c|c|c|c|c|c|c|c|c|}
\hline \multirow[b]{2}{*}{$\rho^{2}$} & \multirow[b]{2}{*}{$l$} & \multicolumn{4}{|c|}{$\operatorname{ARMA}(1,2):\left(\phi_{1}, \theta_{1}, \theta_{2}\right)$} & \multicolumn{4}{|c|}{$\operatorname{ARMA}(2,1):\left(\phi_{1}, \phi_{2}, \theta_{1}\right)$} \\
\hline & & $\begin{array}{c}(-0.8 \\
1.4,-0.6)\end{array}$ & $\begin{array}{l}(0.6 \\
-0.5 \\
-0.9)\end{array}$ & $\begin{array}{c}(0.3, \\
-0.5,0.3)\end{array}$ & $\begin{array}{c}(-0.3 \\
0.3,-0.5)\end{array}$ & $\begin{array}{l}(1.4, \\
-0.6 \\
-0.8)\end{array}$ & $\begin{array}{c}(-0.5 \\
-0.9,0.6)\end{array}$ & $\begin{array}{c}(0.3, \\
-0.5,0.3)\end{array}$ & $\begin{array}{c}(-0.3, \\
0.3,-0.5)\end{array}$ \\
\hline \multirow[t]{4}{*}{0.001} & 1 & 0.0385551 & 0.0049626 & 0.0003949 & 0.0001271 & 0.0373196 & 0.00 & $3.701 \mathrm{e}-05$ & 1.03 \\
\hline & 2 & 0.0125130 & 0.0036421 & $8.082 \mathrm{e}-07$ & $8.818 \mathrm{e}-05$ & 0.0113879 & 0.00 & $3.701 \mathrm{e}-05$ & -06 \\
\hline & 5 & 0.0011102 & $6.436 \mathrm{e}-05$ & $5.878 \mathrm{e}-10$ & $4.681 \mathrm{e}-08$ & 0.0007381 & 0.0002473 & $2.336 \mathrm{e}-06$ & $2.406 \mathrm{e}-07$ \\
\hline & 10 & $9.853 \mathrm{e}-05$ & $3.773 e-07$ & $3.471 \mathrm{e}-15$ & $2.763 \mathrm{e}-13$ & $8.269 \mathrm{e}-05$ & 0.0001450 & $5.223 \mathrm{e}-08$ & $1.047 \mathrm{e}-08$ \\
\hline \multirow[t]{4}{*}{0.01} & 1 & 1.1520600 & 0.2282193 & 0.0334433 & 0.012 & 1.055 & 0.28 & 0.00 & 0.00 \\
\hline & 2 & 0.2772410 & 0.1594559 & $7.032 \mathrm{e}-05$ & 0.0084952 & 0.2756534 & 0.0322743 & 0.0036473 & 0.0006560 \\
\hline & 5 & 0.0245971 & 0.0028177 & $5.115 \mathrm{e}-08$ & $4.509 \mathrm{e}-06$ & 0.0150383 & 0.0202891 & 0.0002308 & $2.377 \mathrm{e}-05$ \\
\hline & 10 & 0.0021831 & $1.654 \mathrm{e}-05$ & $3.020 \mathrm{e}-13$ & $2.662 \mathrm{e}-11$ & 0.0018817 & 0.0106396 & $5.157 \mathrm{e}-06$ & $1.034 \mathrm{e}-06$ \\
\hline \multirow[t]{4}{*}{0.0625} & 1 & 2.8910510 & 0.6771251 & 0.13252727 & 0.0675198 & 2.8509200 & 0.872 & 0.021 & 0.0060423 \\
\hline & 2 & 0.5636513 & 0.4227167 & 0.0003029 & 0.0448998 & 0.6317397 & 0.1399589 & 0.0212131 & 0.0038709 \\
\hline & 5 & 0.0500077 & 0.0074697 & $2.203 \mathrm{e}-07$ & $2.383 \mathrm{e}-05$ & 0.0282102 & 0.0894723 & 0.0013586 & 0.0001398 \\
\hline & 10 & 0.0044384 & $4.384 \mathrm{e}-05$ & $1.300 \mathrm{e}-12$ & $1.407 \mathrm{e}-10$ & 0.0040122 & 0.0409981 & $3.025 \mathrm{e}-05$ & $6.083 \mathrm{e}-06$ \\
\hline
\end{tabular}

Notes:

(1) The observations follow $y_{t}=x_{t}+\varepsilon_{t}$.

(2) For the ARMA $(1,2)$ case, $x_{t}-\phi_{1} x_{t-1}=a_{t}-\theta_{1} a_{t-1}-\theta_{2} a_{t-2}$ with $\sigma_{a}^{2}=1$ and

$$
\sigma_{\varepsilon}^{2}=\rho^{2} \frac{1+\theta_{1}^{2}+\theta_{2}^{2}-\theta_{1} \phi_{1}-\theta_{2} \phi_{1}^{2} \theta_{1} \theta_{2} \phi_{2}}{1-\phi_{1}^{2}} .
$$

(3) For the $\operatorname{ARMA}(2,1)$ case $x_{t}-\phi_{1} x_{t-1}=\phi_{2} x_{t-2}=a_{t}-\theta_{1} a_{t-1}$ with $\sigma_{a}^{2}=1$ and

$$
\sigma_{\varepsilon}^{2}=\rho^{2} \frac{1-\phi_{2}+\theta_{1}^{2}-\phi_{2} \theta_{1}^{2}}{\left(1+\phi_{2}\right)\left(\left(1-\phi_{2}\right)^{2}-\phi_{1}^{2}\right)} .
$$

(3) The result does not depend on the sample size $t$; results shown here are for $t=100$ observations.

and $\left.\theta_{13}\right)$ when the forecast horizon changes from one to two periods. As one would expect, the rate of decrease is smaller for large values of $\rho$.

\section{CONCLUSIONS AND PATHS FOR FURTHER RESEARCH}

In this paper we examined the impact that measurement errors have on prediction generated by ARMA models. Using MMSE and other measures of forecast accuracy we showed that the effect

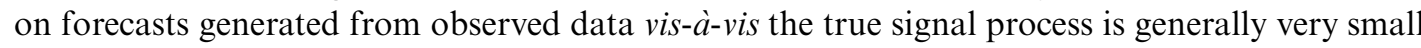
when the noise-to-signal ratio is small and the model parameters are well within the unit circle. The impact, however, can be quite large, particularly for the early forecast horizons, when the parameters are near the unit circle, and they increase in magnitude as the noise-to-signal ratio increases. In these cases it would behoove the model builder to formulate the model in state space form to extract the signal to generate forecasts.

We are currently investigating the possibility of extending our results to non-stationary models. This is mathematically complicated because Theorem 1 and its corollaries are based on the assumption that measurement errors $\varepsilon_{t}$ are uncorrelated in time. If $x_{t}$, and hence, $y_{t}$ are stationary 
after applying the differencing operator $\delta(B)$, the new error term $\delta(B) \varepsilon_{t}$ will be stationary but not uncorrelated. This makes closed forms of $\triangle M M S E$ and $\nabla M M S E$ for $\delta(B) x_{t}$ and $\delta(B) y_{t}$ very difficult, if not impossible, to derive because $R_{t, \delta(B) x_{t}}^{l}$ is not the same as $R_{t, \delta(B) y_{t}}^{l}$, and the difference between $\Gamma_{t, \delta(B) x_{t}}$ and $\Gamma_{t, \delta(B) y_{t}}$ is no longer a diagonal matrix as in equations (11) and (12). Numerical calculations of the mean squared errors for the original, non-stationary series, although tedious, can be made by first obtaining the MMSE's of the stationary series $\delta(B) x_{t}$ and then applying the filter $\delta^{-1}(B)$ to those MMSE's.

It is also interesting to note that extension to non-stationary structures is closely related to models having a stationary signal component and measurement errors that are correlated in time. For example, if the model $y_{t}=x_{t}+\varepsilon_{t}$ is non-stationary, and if taking first differences leads to the stationary model $(1-B) y_{t}=(1-B) x_{t}+(1-B) \varepsilon_{t}$, then $(1-B) y_{t}$ is a combination of a stationary ARMA process with an AR(1) measurement error term. Having more information on different error structures could provide model builders with an even more flexible framework for which to evaluate forecasts.

\section{APPENDIX}

Proof of Theorem 1: Applying Theorem 2.4.7 in Mathai and Provost (1992), we have

$$
\mu_{\text {min }} \mathcal{R}_{t, x}^{l}{ }^{\prime} \Gamma_{t, x}^{-1} \mathcal{R}_{t, x}^{l} \leqslant \mathcal{R}_{t, x}^{l}{ }^{\prime} \Gamma_{t, y}^{-1} \mathcal{R}_{t, x}^{l} \leqslant \mu_{\max } \mathcal{R}_{t, x}^{l}{ }^{\prime} \Gamma_{t, x}^{-1} \mathcal{R}_{t, x}^{l}
$$

where $\mu_{\min }$ and $\mu_{\max }$ are smallest and largest eigenvalues of $\Gamma_{t, x} \Gamma_{t, y}^{-1}$, respectively. Since $\Gamma_{t, x} \Gamma_{t, y}^{-1}=$ $\left(\Gamma_{t, y}-\sigma_{\varepsilon}^{2} I_{t \times t}\right) \Gamma_{t, y}^{-1}=I_{t \times t}-\sigma_{\varepsilon}^{2} \Gamma_{t, y}^{-1}$. Hence we have,

$$
\mu_{\min }=\min _{i}\left(1-\sigma_{\varepsilon}^{2} \frac{1}{\sigma_{\varepsilon}^{2}+\lambda_{i}}\right) \text { and } \mu_{\max }=\max _{i}\left(1-\sigma_{\varepsilon}^{2} \frac{1}{\sigma_{\varepsilon}^{2}+\lambda_{i}}\right)
$$

where $\left\{\lambda_{i}\right\}$ are eigenvalues of $\Gamma_{t, x}$. It is easy to verify that

$$
\mu_{\min }=1-\frac{\sigma_{\varepsilon}^{2}}{\sigma_{\varepsilon}^{2}+\min \lambda_{i}} \text { and } \mu_{\max }=1-\frac{\sigma_{\varepsilon}^{2}}{\sigma_{\varepsilon}^{2}+\max \lambda_{i}}
$$

Replacing $\mu_{\min }$ and $\mu_{\max }$ in equation (A1) by (A2) we obtain

$$
\left(1-\frac{\sigma_{\varepsilon}^{2}}{\sigma_{\varepsilon}^{2}+\min _{i} \lambda_{i}}\right) \mathcal{R}_{t, x}^{l}{ }^{\prime} \Gamma_{t, x}^{-1} \mathcal{R}_{t, x}^{l} \leqslant \mathcal{R}_{t, x}^{l}{ }^{\prime} \Gamma_{t, y}^{-1} \mathcal{R}_{t, x}^{l} \leqslant\left(1-\frac{\sigma_{\varepsilon}^{2}}{\sigma_{\varepsilon}^{2}+\max _{i} \lambda_{i}}\right) \mathcal{R}_{t, x}^{l}{ }^{\prime} \Gamma_{t, x}^{-1} \mathcal{R}_{t, x}^{l}
$$

or

$$
\frac{\sigma_{\varepsilon}^{2}}{\sigma_{\varepsilon}^{2}+\max _{i} \lambda_{i}} \mathcal{R}_{t, x}^{l}{ }^{\prime} \Gamma_{t, x}^{-1} \mathcal{R}_{t, x}^{l} \leqslant \Delta M M S E \leqslant \frac{\sigma_{\varepsilon}^{2}}{\sigma_{\varepsilon}^{2}+\min _{i} \lambda_{i}} \mathcal{R}_{t, x}^{l{ }^{\prime}} \Gamma_{t, x}^{-1} \mathcal{R}_{t, x}^{l}
$$

If we let

$$
B_{1, t} \equiv \gamma_{x}(0)-\operatorname{MMSE}(\hat{x}(l)) \equiv \mathcal{R}_{t, x}^{l}{ }^{\prime} \gamma_{t, x}^{-1} \mathcal{R}_{t, x}^{l}
$$


and

$$
B_{2, t} \equiv\left[\gamma_{x}(0)-\operatorname{MMSE}(\hat{x}(l))\right] / \operatorname{MMSE}(\hat{x}(l))
$$

we obtain the two inequalities for $\triangle M M S E$ and $\nabla M M S E$ of Theorem 1.

\section{ACKNOWLEDGEMENTS}

We are grateful to William R. Bell for useful comments on the early version of the paper.

\section{REFERENCES}

Bell, W. and Wilcox, D., 'The effect of sampling error on the time series behavior of consumption data', Journal of Econometrics, 55 (1993), 235-65.

Bell, W. and Hilmer, S., 'The time series approach to estimation for repeated surveys', Survey Methodology, 16 (1990), 195-215.

Box, G. E. P. and Jenkins, G. M., Time Series Analysis: Forecasting and Control, San Francisco: HoldenDay, 1976.

Brockwell, P. and Davis, R., Time Series: Theory and Methods, New York: Springer-Verlag, 1991.

Granger, C. W. J. and Morris, M. J., 'Time series modelling and interpretation', Journal of the Royal Statistical Society, Series A, 139 (1976), 246-57.

Gregory, R. and Karney, D., A Collection of Matrices for Testing Computational Algorithms, New York: Wiley-Interscience, 1969.

Grenander, U. and Szegö, G., Toeplitz Forms and their Applications, New York: Chelsea Publishing Company, 1958.

Kron, G. and Kron, T., Mathematical Handbook for Scientists and Engineers, New York: McGraw-Hill, 1961.

Mathai, A. M. and Provost, S. B., Quadratic Forms in Random Variables, New York: Marcel Dekker, 1992.

Pukkila, T., Koreisha, S. and Kallinen, A., 'The identification of ARMA models', Biometrika, 77 (1990), 537-48.

Wilcox, D., 'The construction of U.S. consumption data: some facts and their implications for empirical work', The American Economic Review, 82 (1992), 922-41.

Wong, W.-K. and Miller, R. B., 'Repeated time series analysis of ARIMA-noise models', Journal of Business \& Economic Statistics, 8 (1990), 243-50.

Authors' biographies:

Sergio A. Koreisha is the Charles H. Lundquist Research Scholar and Professor of Decision Sciences at the Lundquist College of Business, University of Oregon. He specializes in the areas of forecasting, mathematical model building, applied econometrics, production analysis, and manufacturing strategy. Some of his latest works in the field of forecasting include articles dealing with new fast estimation methods for large-scale multiple-time series models; new approaches for identifying the mathematical structures governing the behavior of economic time series; and how causality among economic and business variables might be ascertained and used to formulate more accurate models. He has taught courses in Europe, South America, and the Pacific. He also provides consulting services for companies interested in doing business in Brazil.

Yue Fang is assistant professor of Decision Sciences at the Lundquist College of Business, University of Oregon. He has a Ph.D. in Statistics from MIT. His publications include articles on financial econometrics, time series forecasting and statistical quality controls. His recent work focuses on the quantitative analysis of financial markets using mathematical and statistical models.

Authors' address:

Sergio A. Koreisha and Yue Fang, Lundquist College of Business, University of Oregon, Eugene, OR 97403, USA. 